\title{
Universidad Autónoma de Chihuahua, el contexto durante su creación en 1954
}

\author{
Autonomous University of Chihuahua, the context during its \\ creation in 1954
}

\author{
Guillermo Hernández Orozco* \\ Stefany Liddiard Cárdenas** \\ Francisco Alberto Pérez Piñón ${ }^{* * * *}$
}

\begin{abstract}
* Profesor de la Universidad Autónoma de Chihuahua (México). Es doctor en Ciencias de la Educación por el Instituto Superior Pedagógico Enrique José E. Varona (Cuba). Entre sus publicaciones recientes están: "José Joaquín Calvo López, fundador del Instituto Cientifico y Literario, hoy Universidad Autónoma de Chihuahua" en IE Revista de Investigación Educativa, Vol. 8, No. 14, (2017) e Historia a Debate (Cinco Tomos). Obtuvo el reconocimiento Eduardo Flores Kastanis a la Investigación Educativa 2018. Cuenta con reconocimientos al Perfil Prodep y del Sistema Nacional de Investigadores, nivel 1. Sus temas de interés son la historia e historiografia de la educación. Correo electrónico ghernand@uach.mx
\end{abstract}

http://orcid.org/0000-0001-7287-8240

\begin{abstract}
**Profesora de la Universidad de Ciencias del Comportamiento (México). Es Doctora por la Universidad Autónoma de Chihuahua y maestra en Psicología Social y de las Organizaciones. Entre sus publicaciones recientes están: "La hacienda de Humboldt en Chihuahua, lugar de refugio para los bóers precedentes de Sudáfrica a principios del siglo XX”, en Espacio abierto: cuaderno venezolano de sociología, Vol. 28, No.1, (2019); Fundamentos teóricometodológicos en la investigación educativa en Chihuahua; análisis de un área del conocimiento", en IE Revista de Investigación Educativa de la REDIECH, Vol.10, No. 18, (2019). Es socia activa de la Red de Investigadores Educativos Chihuahua y de la Sociedad Mexicana de Historia de la Educación. Sus temas de interés son la historia e historiografia de la educación. Correo electrónico stefanyliddiard@gmail.com
\end{abstract}

(i) https://orcid.org/0000-0002-3234-4372

**Profesor investigador de la Universidad Autónoma de Chihuahua (México). Es doctor en Ciencias Pedagógicas (Cuba). Entre sus publicaciones recientes están: “Apuntes para una historia posmodernista” en Espacio abierto vol. 25, n.4 (2016); capitulo de libro "La hacienda de coyotillos, un acercamiento a los contenidos educativos", en Acercamientos a la historia de la educación, diálogos actores y fuentes en la construcción del conocimiento histórico (2017). Desarrolla la línea de investigación Historia e Historiografía de la Educación. Cuenta con reconocimientos Prodep y del Sistema Nacional de Investigadores, nivel 1. Es miembro de la Sociedad Mexicana de Historia de la Educación, del Consejo Mexicano de Investigación Educativa y de la Red de Investigadores Educativos Chihuahua. Correo electrónico aperezp@uach.mx

https://orcid.org/0000-0003-4316-6484

Historial editorial

Recibido: 07-febrero-2019

Aceptado: 22-agosto-2019

Publicado: 23-septiembre-2019

ISSN-e: 2594-2956 


\section{Universidad Autónoma de Chihuahua, el contexto durante su creación en 1954}

\section{Resumen}

En este trabajo se pone de relieve el contexto en que nace la actual Universidad Autónoma de Chihuahua, de las condiciones que pocas veces se toman en cuenta cuando se habla del surgimiento de instituciones. Desde esa perspectiva se muestran algunos datos estadísticos, poblacionales, económicos y educativos de la entidad en torno a I954; además de esto, como parte de ese mismo contexto, se documentan los antecedentes de la creación de la universidad en México, así como los precedentes en Chihuahua, con la creación del Instituto Científico y Literario. Basta decir que la Universidad Autónoma de Chihuahua no surge de la nada, ni de personas iluminadas, sino que ya existían ciertas condiciones poblacionales, educativas, ocupacionales y culturales para transformar el Instituto Científico y Literario en una universidad. Sobre este último punto, se muestran los programas académicos iniciales, además de mencionar a directivos, personal académico y alumnos, quienes emprendieron el nuevo camino de formación de recursos humanos profesionales acordes y condicionados por la realidad.

Palabras clave: Historia de la educación, instituciones educativas, Universidad. 
Autonomous University of Chihuahua, the context during its creation in 1954

\begin{abstract}
This paper highlights the context of the creation of the Autonomous University of Chihuahua, of the conditions that are rarely taken into consideration when talking about the emergence of institutions. From this perspective, statistical data of population, economy and education of this state around 1954 are shown. Moreover, as part of that same context, background information is documented regarding the creation of the university in Mexico and the creation of the Scientific and Literary Institute in Chihuahua. This university did not emerge out of nowhere, nor from enlightened people, but from the already existent conditions of population, education, occupation and culture that were needed to transform the Scientific and Literary Institute into a university. Regarding this last point, the initial academic programs are shown, in addition to mentioning executives, academic staff and students, who undertook the new path of training professional human resources according to and conditioned by reality.
\end{abstract}

Keywords: history of education, educational institutions, university. 
Université autonome de Chihuahua, contexte lors de sa création en 1954

\section{Résumé}

Ce travail met en évidence le contexte dans lequel est née l'Université autonome de Chihuahua actuelle, des conditions qui sont rarement prises en compte lorsqu'on parle de l'émergence d'institutions. Dans cette perspective, quelques données statistiques, démographiques, économiques et éducatives de l'entité vers 1954 sont présentées; En plus de cela, dans le même contexte, les antécédents de la création de l'université au Mexique, ainsi que les précédents à Chihuahua, sont documentés avec la création de l'Institut scientifique et littéraire. Il suffit de dire que l'Université autonome de Chihuahua n'est pas née de nulle part, ni de personnes éclairées, mais qu'il existait déjà certaines conditions de population, d'éducation, d'occupation et de culture pour transformer l'Institut scientifique et littéraire en université. Sur ce dernier point, les programmes académiques initiaux sont présentés, en plus de mentionner les dirigeants, le personnel académique et les étudiants, qui ont entrepris la nouvelle voie de la formation des ressources humaines professionnelles selon et conditionnés par la réalité.

Mots-clés: Établissements d'enseignement, Histoire de l'éducation, Université. 
Universidad Autónoma de Chihuahua w kontekscie lat piedziesiatych, w momencie jego powstania

Streszczenie Ta praca podkreśla kontekst, w którym rodzi się obecny uniwersytet w Chihuahua. Zostaly zanalizowane niektóre dane statystyczne, demograficzne, gospodarcze i oswiatowe pochodzace z I954 roku; oprócz tego, wzięte zostalo pod uwagę ogólne tło, ktore przyczynilo się do tworzenia uniwersytetów w Meksyku, jak również w Chihuahua, i które są udokumentowane, wraz z utworzeniem Instytutu Naukowego i Literackiego, w wymienionym miescie. Wystarczy powiedzieć, że ewolucja Instytutu Nukowego i Literackiego i przeksztalcenie go w uniwersytet w Chihuahua bylo wynikiem róznych ruchów akdemickich i intelektualnych. W tekscie zaanalizowane zostaly początkowe programy akademickie, wspominania menedżerów, kadry akademickiej i studentów, którzy zapoczatkowali historie oswiaty uniwersyteckiej w Chihuahua.

\section{Slowa kluczowe:}

Historia oswiaty, instytucje pedagogiczne, uniwersytet. 


\section{Introducción}

Cuando se hace historia e historiografía, emerge uno de los problemas más difíciles de dilucidar: reconstruir el contexto que rodea a él o los hechos que se buscan conocer, analizar y en todo caso, interpretar. Se trata de conocer las situaciones que inciden sobre dichos eventos y es aquí que aparecen los obstáculos que consisten en juzgar desde la cultura actual, con las teorías y al lado de las experiencias de quien hace esta historia, para identificar las condiciones de hechos pasados hace uno o dos siglos. Esta estructura interpretativa de las personas se construye a lo largo de la vida y tiene diversos componentes como la familia donde se nace, la o las comunidades donde ha vivido, la formación académica, los puestos o labores desempeñadas y las condiciones vividas: económicas, psicológicas, políticas, sociales y en todo caso culturales. Es dentro de ese proceso que se conforman utopías, esperanzas, desesperanzas y contradicciones en las interacciones de esa vida cotidiana y visión del futuro.

Entonces, es común leer la historia en la cual se explican una serie de sucesos, pero en la que se elimina la complejidad de las diversas variables que acontecen; en la mayoría de las ocasiones, atribuyéndola a ciertos héroes y villanos, a personas ilustres, iluminadas, valientes a toda prueba y prácticamente solos en su quehacer. Una historia de aquellos que con su arrojo realizan revoluciones y transformaciones que trascienden en el tiempo. Pero hay que preguntarse ¿en qué medida

192 son los contextos económicos, políticos, culturales y sociales los principales protagonistas de la historia?

Este trabajo tiene como finalidad poner de manifiesto ese contexto que incidió al momento de crear una institución educativa, visto desde diversas aristas, con el propósito de tener una visión más completa, que 
claro, no está exenta de ausencias. En el caso concreto del surgimiento de la Universidad de Chihuahua en 1954, resulta beneficioso, ya que para quienes no lo vivieron, resultaría difícil imaginar cuáles fueron los contextos nacionales y estatales que rodearon la creación de esta institución.

En ese tenor se presenta este texto, fundamentado en el paradigma socio-histórico sobre el cual se reconstruye la historia de una institución educativa. Para lograrlo fue necesario recuperar tanto de fuentes primarias, como secundarias, la información necesaria para describir este contexto. Se revisaron estadísticas, censos, libros que abordan la temática y entrevistas con personajes significativos que fueron parte importante de esta reconstrucción histórica, social y contextual del origen de la Universidad de Chihuahua. Derivado de lo anterior es que se organiza este trabajo en tres partes. En la primera se muestran los datos estadísticos, demográficos, geográficos y educativos que rodearon el escenario social de esta incipiente institución, durante la década de 1950. En una segunda parte se describe la matrícula, instalaciones y personajes del Instituto Científico y Literario, que se transformaría en Universidad de Chihuahua en 1954. Finalmente, en el tercer apartado se muestran datos y fragmentos derivados de las comunicaciones con algunos de los maestros fundadores de esta institución.

\section{Los hilos del entramado de Chihuahua: 1954}

Este apartado comienza con el análisis de la pertinencia de las carreras que se ofertaban al inicio de la Universidad de Chihuahua, para lo cual se recuperan datos estadísticos de los años cincuenta, principalmente de tres fuentes de información: Banco Nacional Hipotecario Urbano y 
de Obras Públicas (I959), censo de población de 1950 que emitió el Instituto Nacional de Estadística y Geografía (INEGI, I950) y libro con la compilación de informes en el quincuagésimo aniversario de la Universidad Autónoma de Chihuahua (Hernández, 2004). Con toda esta información se dedujo la justificación que hubo en aquel momento para establecer ciertas instituciones de educación superior, su ubicación, las carreras que se ofertaban e incluso el campo de aplicación de los estudios profesionales.

Según los informes descritos en el censo de población que dio a conocer el INEGI en I950, la población del estado de Chihuahua era de 846 mil 423 personas y los municipios que tenían la mayor población era Juárez con el $15.5 \%$ del total, Chihuahua $13.3 \%$, Parral $4.3 \%$, Delicias y Cuauhtémoc 3.6\% y Guerrero 3\%. Con estos datos se observa que los seis municipios mencionados concentraban el $46.3 \%$ de la población; considerando que para entonces el estado estaba compuesto por 65 municipios y no por 67 como ocurre en la actualidad. Con relación al porcentaje de población urbana en cada municipio, Juárez contaba con el 93.3\%, Chihuahua 77.4\%, Santa Bárbara 77.I\%, Camargo 64.2\%, y Delicias y San Francisco del Oro 59.7\% cada uno. Al estar en un territorio mayoritariamente desértico, la población que se conglomeraba en los principales centros urbanos permaneció sin mucha variación durante varias décadas.

En cuanto a la extensión territorial hay que hacer notar que Ahumada 194 cuenta con el $7.3 \%$ de la superficie estatal, Chihuahua 5.2\%, Buenaventura 4.8\%, Ojinaga 4.5\%, y Guadalupe y Calvo 4.I \%. Correspondiente con el tamaño y población en los diversos centros urbanos, se encontraban también los servicios, entre los cuales se contemplaron los educativos. Aunque Ciudad Juárez era el municipio con mayor población, fue en Chihuahua donde se creó la primera 
universidad, hecho que los habitantes de la frontera fueron reclamando, cada año con más fuerza, logrando hasta 1973 crear su propia institución, la Universidad Autónoma de Ciudad Juárez (UACJ). Esto hizo que Chihuahua fuera uno de los pocos estados que cuentan con dos universidades autónomas públicas.

Con este primer acercamiento es posible comprender acerca de los aspectos demográficos y geográficos que incidieron en el proceso de conformación de las universidades estatales en México, particularmente en Chihuahua. Es por ello que se debe cuestionar el por qué se inauguró en Chihuahua la primera universidad y tardíamente en Ciudad Juárez, si la concentración de la población que demandaba estudios superiores no se encontraba en la capital. También es necesario considerar las condiciones necesarias para que las universidades públicas ganaran la autonomía, pues la Universidad de Chihuahua se creó en I954 pero no fue hasta I968, mediante diversas gestiones, que obtuvo su autonomía, a diferencia de la UACJ que nació ya con ese distintivo.

Además de la información anterior, existen otros factores que influyeron en el desarrollo contextual de la Universidad, los cuales también se recuperan del censo ya mencionado. En este caso se puso interés en las ocupaciones de los chihuahuenses en la década de los años cincuenta, que mayoritariamente se dedicaban a la agricultura, silvicultura, caza y pesca, que en conjunto agrupaban el $55 \%$ de las actividades productivas. Por su parte, la obtención de recursos económicos para el estado provenía principalmente de la explotación de los metales. Chihuahua era un estado minero, siendo una ocupación sobresaliente que aportaba un $55 \%$ del ingreso estatal, seguida por la recaudación de impuestos con el $6.2 \%$, en tanto que los derivados del tabaco y las bebidas embriagantes sumaban el $4.8 \%$. En cuanto al valor 
de la ganadería, el vacuno mantuvo el $66.8 \%$ del valor del total del área, el caballar 6.2\%, los animales de trabajo con el $\mathbf{1 2 . 4 \%}$, y finalmente el porcino con el 4.I\%. Con estos datos se pone de relieve que Chihuahua en esa década era considerado un estado agrícola y ganadero, principalmente.

En cuanto al comercio, en Ciudad Juárez se reunía el $48.2 \%$ de los principales ingresos, Chihuahua congregaba el I8.6\%, Parral 5\%, Delicias $3.3 \%$ y Guadalupe y Calvo o.I \%. En lo concerniente a la producción agrícola y frutícola estatal, el algodón ocupaba el 62.6\%, maíz I6.I\%, avena $6 \%$, trigo $3.9 \%$, manzana $0.8 \%$ y durazno $0.3 \%$. En aquel momento se conocía como la época del oro blanco -cultivo del algodón- al que se le apostaron muchos recursos, pero lamentablemente pronto entraría en crisis. En contraste, la manzana aparecía al final del listado, pero se convirtió en una fuente relevante de producción en las siguientes décadas.

\section{Datos estadísticos en la educación}

Fuera del contexto estatal y pasando a nivel nacional, se trae a colación que fue en 1553 cuando se creó la primera universidad en México, misma que fue suprimida en I833, reabriendo sus puertas hasta I9Io. Después de ese momento, existió un lapso de cincuenta años durante los cuales se fueron transformando las Instituciones de Educación Superior de los diversos estados.

Durante el siglo XIX se conformó el sistema educativo mexicano, sobre todo a partir de la primera Ley Orgánica en I867. En ese momento se pretendía avanzar de un periodo de ilustración hacia el positivismo, 
teniendo su expresión más sobresaliente en el campo educativo, durante el Congreso Nacional de Educación celebrado entre I889 y I890. En este evento sentaron las bases teóricas para que toda la nación caminara hacia la ciencia y su aplicación.

En Chihuahua, durante ese mismo siglo, se creó el Instituto Literario en I835, añadiéndole a su nombre el adjetivo de científico en I88I y se mantuvo de esa forma hasta I954, cuando se transformó en universidad. El contexto de creación de la máxima casa de estudios de Chihuahua en 1954- también posee estos hilos del entramado, que en gran medida ayudan a comprender las condiciones del surgimiento. Para ello es conveniente rescatar algunos datos que publicó la Universidad Nacional Autónoma de México (UNAM) en I959, en los cuales según se asientan en el volumen de estadística, dentro de su matrícula, existían estudiantes provenientes del estado de Chihuahua.

En aquel momento la UNAM contaba con 33 mil 428 estudiantes, de los cuales Io mil 398 eran de preparatoria y el resto de estudios profesionales, es decir 23 mil 030. De ese total, 28 mil o48 eran hombres y 5 mil 380 mujeres, hecho que deja ver que existían oportunidades de estudios para mujeres, con aproximadamente el quince por ciento de la matrícula. En comparativa, cuando inició el año escolar en la Universidad de Chihuahua, la matrícula registrada fue de 39 estudiantes en las tres carreras profesionales que se ofertaron: Ingeniería Civil, Derecho y Medicina. De ese total solo ingresó una mujer en la última carrera.

En los datos estadísticos de la UNAM (1959) se muestra que en 1958 (porque no se dispone de datos de I955) se matricularon I07 estudiantes provenientes de Chihuahua, de los cuales 23 estaban registrados en Preparatoria, I4 en Arquitectura, uno en Artes Plásticas, tres en el área 
de ciencias (Biología, Física y Matemáticas), tres en la Escuela Nacional de Ciencias Políticas y Sociales, II en Contaduría y Administración, i8 en Derecho, tres en Economía, seis Filosofía, ocho en Ingeniería, tres en Medicina, uno en Odontología, tres en Ciencias Químicas y uno en Veterinaria.

Debido a la cantidad de estudiantes que migraban a aquella institución de nivel superior de la Ciudad de México, durante el primer consejo universitario de la naciente Universidad de Chihuahua, se tomó la decisión de signar un acuerdo, especificando que la UNAM no debería aceptar a estudiantes provenientes de Chihuahua en aquellas carreras que ya existían en la entidad. En ese momento la suma de estudiantes inscritos en la UNAM, oriundos del estado y que podían estar estudiando en la Universidad de Chihuahua, era de 29 (I8 para la Licenciatura en Derecho, ocho en Ingeniería y tres en Medicina.

En el momento que se creó la Universidad de Chihuahua, eran 24 las instituciones que integraban la Asociación Nacional de Universidades e Institutos de Educación Superior (ANUIES). Como dato adicional es conveniente señalar que esa denominación al paso del tiempo cambió de institutos a instituciones, ya que en esa época aun existían los llamados Institutos Científicos y Literarios en algunos estados: Aguascalientes, Chiapas, Hidalgo, Estado de México, Oaxaca, Zacatecas y Chihuahua.

198 El Instituto Científico y Literario de Chihuahua (ICL), según menciona Hernández (1998), se fundó el I9 de marzo de I835 en la que fuera casa habitación de Mariano Horcasitas, ubicada entre las actuales calles Victoria y $4^{\text {a }}$ en el centro de la capital de Chihuahua, lugar que ahora funciona como estacionamiento del Hotel San Francisco. En 1954, el ICL cerró un ciclo de II9 años y se transformó en universidad. Las 
nuevas carreras como Medicina, Derecho e Ingeniería se adjuntaron a la Escuela de Farmacia, Escuelas Prevocacionales -equivalente a secundaria técnica-, la Vocacional -equivalente a la preparatoria técnica- y la Preparatoria. Además, otras instituciones fueron integradas a la naciente Universidad, tal fue el caso de Enfermería y Bellas Artes.

Sintetizando algunos datos, fue el 3 de enero 1955 cuando se inauguraron las clases en el lugar conocido como Paraninfo, la matrícula en aquel momento fue de 235 estudiantes en Pre-vocacional, 443 en Preparatoria, I86 en Farmacia, I3 en Ingeniería, I2 en Derecho, 15 en Medicina y 69 en el incipiente Instituto de Bellas Artes. La suma daba 973 estudiantes (Hernández, I998).

Es necesario tener presente que en aquel momento el contexto político era polémico, pues el gobernador en turno, Lic. Oscar Soto Máynez, después de la inauguración de la Universidad tuvo que salir por la puerta lateral ya que, a unas cuadras de distancia en la Plaza Hidalgo, lo esperaba una multitud que lo acusaba de ser el autor intelectual del asesinato del taxista Cereceres. Esta anécdota la comentó Humberto Hinojos Aranda, quien fue el representante del municipio de Ojinaga y posteriormente director de la Facultad de Ingeniería y Secretario Académico de la Universidad Autónoma de Chihuahua.

En ese año relevante para la Universidad de Chihuahua (I955), los funcionarios iniciadores fueron el Dr. Ignacio González Estavillo como Rector, el Dr. Julio Ornelas K. como director de la escuela de medicina, el Ing. Federico Pérez Márquez al frente de la Escuela de Ingeniería, el Lic. Felipe Lugo Fernández en la Escuela de Derecho, el profesor Ernesto Talavera en la Escuela de Música, el Dr. Enrique de Noriega en la Escuela de Enfermería y Obstetricia y el Dr. Rogelio Murillo en la 
Escuela Preparatoria (Hernández, I998). En este primer listado se hace notar que no aparece el director de Farmacia y que cuatro funcionarios eran médicos, uno ingeniero, un abogado y un profesor. La naciente institución era territorio primordialmente de médicos de profesión.

Hernández (1998) menciona, en cuanto a la primera planta académica en I955, que en la Escuela de Ingeniería todos los docentes contaban con el título afín, entre los que estaban Jesús Roberto Durán Gutiérrez, Alberto Enríquez Muños, Alfonso Jurado, Héctor García Terrazas, José de la Luz González, José Medrano Morales, Federico Pérez Márquez y Eduardo Prado Rodríguez. En la Escuela de Medicina todos los docentes contaban con el perfil de médicos. Entre ellos estaban Gilberto Pérez Rodríguez, Jesús Gómez Cruz, Carlos Aguirre, Francisco Uranga Vallarta, Fernando Martínez López y Ángel Abbud Ochoa. Por su parte, en la Escuela de Derecho iniciaron los licenciados Óscar Ornelas Kuchle y Mario F. García. Finalmente, los académicos de la Escuela de Farmacia fueron el químico Samuel Bencomo Lasso, los profesores Jesús Grajeda Pedrueza, Alfonso Luján, Pedro Gómez Rodríguez, los ingenieros Héctor P. García y Luis G. Romero Molina, la señorita Carmen Terrazas, el licenciado Manuel O`Reilley y la profesora Celedonia González. Cabe mencionar que esta última escuela se encontraba en labores desde 1942 dentro del Instituto Científico y Literario.

Ahora bien, referido a las instalaciones en donde principió la 200 Universidad de Chihuahua fueron las ya existentes del Instituto Científico y Literario, lo que actualmente es la Rectoría. En ese lugar funcionaban la Escuela de Farmacia, las escuelas Secundaria y la Preparatoria, pero llegó el momento que resultaron insuficientes. Ante esta demanda de espacios físicos, se ubicó a la Escuela de Ingeniería en la Quinta Gameros; en el Hospital Central Universitario a la Escuela de 
Medicina y Enfermería; y el Instituto de Bellas Artes inició labores en una casa habitación ubicada en la calle segunda en el centro de la ciudad, pero en corto tiempo se trasladó también a la Quinta Gameros (Hernández, I998).

Así mismo, en lo que actualmente es el Campus I de la Universidad, se colocó la primera piedra el día 6 de mayo de i955. Allí fue el cementerio Santo Niño, según lo relata Amanda Hernández (1986) en el libro que escribió acerca del General Miguel Saavedra Romero. Este dato se corrobora porque actualmente se conserva el arco que daba entrada al cementerio, es triple y se encuentra en el camellón intermedio, casi frente a la actual Facultad de Artes. Los edificios que originalmente se construyeron con fines universitarios fueron: Derecho -lo que hoy es la Facultad de Filosofía y Letras-, la Preparatoria -hoy Facultad de Derecho-, la Escuela Normal -hoy Facultad de Derecho- y Ganadería, que hoy funcionan como instalaciones administrativas frente a la Ciudad Deportiva.

\section{Conversaciones con maestros fundadores}

Sin precisar la fecha de cada una, a continuación se muestran algunos datos recabados de entrevistas con maestros fundadores, entre quienes se encuentran Felipe Salas Plata, José Velasco Ortiz, Héctor García Campos, Julio Ornelas Kuchle, Luz Hayashi, María Cervantes, Dolores Delgado, Carlos García Gutiérrez, Luján Sánchez, Benito Nogueira y José Medrano Morales.

En la plática con Felipe Salas Plata menciona que inició labores en el Instituto Científico y Literario en 1948 y continúa hasta 1955. Señala que 
compartió con dos personajes que consideró importantes: Hipólito Vela y Pedro Barba, quienes fueron puntales en la creación de la Escuela de Educación Física en 1956.

Otro maestro fundador fue José Velasco Ortiz, quien ingresó en 1950 y trabajó hasta 1955. Cabe mencionar que esta labor la desempeñó gratuitamente, como una forma de retribuir al Instituto Científico y Literario los cinco años que estudió en el plantel. Su función en ese periodo fue como maestro de biología y propedéutica médica.

Por su parte, Héctor García Campos era recién egresado de la Escuela de Farmacia cuando inició labores en 1945, siendo paralelamente profesor y secretario de esa escuela. La familia de Héctor también estuvo cercana al Instituto Científico y Literario, ya que su padre Juan García fue director en el periodo I9I4 a 1920; su hermana Bertha García entró al Instituto cuando tenía I2 años, estudió y tiempo más tarde fue maestra en San Carlos, en la Cuidad de México. En I992 fue cuando se pudo entrevistar a Héctor García, cuando se jubiló y regresó a Chihuahua.

Julio Ornelas Kuchle fue fundador y también estudió en el antiguo Instituto Científico y Literario, donde fundó la cátedra de higiene de la juventud. Afirmó durante la conversación que Martín H. Barrios Álvarez fue el principal impulsor de la creación de la Universidad. Julio Ornelas fungió como consejero de gobernadores y aseguró que en el 202 lecho de muerte le dijo al gobernador Soto Máynez, "si te quieres inmortalizar, funda la Universidad”. Fue Ornelas quien pronunció el discurso oficial de apertura de la Universidad y también se desempeñó como director del Instituto Científico y Literario, separándolo en 1937 de la Escuela Normal. 
Luz Hayashi fue docente de la Escuela Preparatoria. En 1939 se tituló como profesora e inició en 1949 su trabajo en el Instituto Científico y Literario. Durante la entrevista afirmó que los maestros eran de planta y debían prepararse "para hacer lo que fuera”. Comentó además que fue Martín H. Barrios Álvarez quien la recomendó para que fuera maestra en el Instituto Científico y Literario. Años más tarde se jubiló como profesora de la Escuela Secundaria Técnica No. 2 ETIC-Ioo, cuando la escuela pre-vocacional pasó a ser Instituto Tecnológico Regional y luego se convirtió en dicha Secundaria Técnica. Estando en ese puesto recibió la medalla Altamirano a los 43 años de servicio y cabe mencionar que su hermana y hermano también fueron maestros del Instituto Científico y Literario.

María Cervantes se formó como profesora normalista, trabajó en la Escuela de Farmacia desde I946, en donde impartía la asignatura de botánica. Menciona que el profesor Jesús Grajeda Pedrueza fue quien le asignó dichas clases, en el momento que concibe como "el tiempo en que había más respeto por los profesores".

Corría el año 1949 cuando otra de las maestras empezó a trabajar en el Instituto Científico y Literario. Se trataba de Dolores Delgado, quien fue llamada por el profesor Jesús Grajeda Pedrueza, entonces secretario de la Preparatoria. Dolores mencionó en la comunicación que "era época difícil, sobre todo cuando nos cambiamos a batallar a la nueva Ciudad Universitaria, en I955, porque estaba muy lejos”.

El ingeniero Carlos García Gutiérrez ingresó en octubre de 1954, dos meses antes del decreto de creación de la Universidad. Se encargó del área de recursos minerales y dispuso para ello un espacio físico en la Quinta Gameros. Señala que en aquel momento solamente tenía ocho 
alumnos. En este mismo ámbito hay que resaltar que posteriormente fue el creador de la carrera de Geología.

En otro momento se conversó con el ingeniero Luján Sánchez, quien nació en Villa López y estudió su educación primaria en Jiménez. La secundaria la cursó en el Instituto Científico y Literario, ingresando en 1927. Narra que cuando se fue a México a estudiar ingeniería, él trabajaba y estudiaba al mismo tiempo, por lo cual la maestra Carlota Maceyra lo sustituyó en sus labores. Fue en 1938 que reinició actividades en el Instituto Científico y Literario. Afirmó que fue el primer profesionista de Villa López y que al impartir botánica fue adoptando los contenidos de una revista argentina. También mencionó que una de sus maestras que recordaba fue Bertha García. Luján fue maestro fundador de la Escuela de Medicina e indica que "en mi tiempo de maestro, el Congreso del Estado aprobó un programa para titular a los egresados de Farmacia”. También fundó la Escuela de Pre-medicina destinada para aquellos que no lograban entrar con el primer examen a la carrera, contabilizando una inscripción en la primera generación de cincuenta estudiantes.

En cuanto a Benito Nogueira menciona que en 1948 se inició como maestro en el Instituto Científico y Literario como docente del curso de física en la Escuela de Farmacia. Fue hasta 1953 que se incorporó al Instituto de Bellas Artes, durante la administración de José Fuentes Mares como Rector de la Universidad.

Finalmente, José Medrano menciona que tuvo como profesores a Enrique Müller, quien fuera fundador de la Escuela de Ingenieros Mecánicos y Electricistas (ESIME) en 1934, como parte del Instituto Científico y Literario, dos años antes de que se fundaran esos estudios en la Ciudad de México, en el Instituto Politécnico Nacional. Asimismo, 
menciona que fue Roberto Ornelas K., quien le cedió su cátedra en el Instituto Científico y Literario, ya que Ornelas se dedicó de tiempo completo al Instituto Tecnológico Regional de Chihuahua, del que fue fundador en 1948. Se menciona también que José Medrano fue secretario de Manuel López Dávila, cuando este último fue el responsable de la implantación del socialismo en Chihuahua. López Dávila posteriormente se desempeñó como gobernador de San Luis Potosí y trabajó cercanamente con el presidente de México Adolfo López Mateos. No se cierra la conversación sin señalar que José Medrano también fue presidente de la Sociedad Chihuahuense de Estudios Históricos.

\section{Conclusiones}

Después de mostrar todos estos elementos, se concluye que los contextos son determinantes cuando se trata del surgimiento de instituciones educativas, como fue el caso de la Universidad de Chihuahua, ya que poco a poco se fueron dando las condiciones para su creación. También se rescató que el Instituto Científico y Literario se modernizó lentamente, creando nuevas escuelas en diferentes momentos, que dieron respuesta a los requerimientos sociales de la época: Escuela Comercial, Escuela Normal, Escuela de Ingenieros Mecánicos y Electricistas, Escuela de Farmacia y sobre todo una base cuantitativamente grande en lo que era la Preparatoria, requisito indispensable para ingresar a estudios universitarios.

Se infiere que las demandas de los padres de familia que -por su condición económica- no podían enviar a sus hijos a estudiar fuera de Chihuahua, constituyeron un impulso y soporte social para la creación 
de las Instituciones de Educación Superior. Para aquel momento cada estado de la República conformaba su propia universidad, la cual tendría el carácter de pública y serviría para toda la entidad, por esta razón se llamó Universidad de Chihuahua, entendiendo que daría servicio a las peticiones a lo largo del estado, aunque las escuelas preparatorias solo había en Parral, Ciudad Juárez y Chihuahua.

Se concluye también que las condiciones económicas en ese momento de la historia -y en Chihuahua- requerían cierta formación de profesionales que pudieran solventar los empleos demandados y las nuevas necesidades en las empresas agrícolas y comerciales. El aparato gubernamental necesitaba abogados, el sector salud médicos y farmacéuticos, el crecimiento de las ciudades requería ingenieros, y el sector agrícola y ganadero también reclamaría ciertos perfiles de profesionistas.

Ciertamente se asegura que la combinación de maestros, políticos, y comerciantes que decidieron crear la Universidad de Chihuahua en I954, conformaban una masa crítica de profesionistas que también fungieron como maestros. Pero las cuestiones que determinaron dicha creación fue principalmente el contexto, que jugó el papel principal y para ello, al pasar el tiempo, los números son buenos protagonistas para entenderlos.

\section{Referencias}

Banco Nacional Hipotecario Urbano y Obras Públicas (1959). Obras y Servicios Públicos. Estado de Chihuahua. México: BANOBRAS. 
Hernández, A. (1986). General Miguel Saavedra Romero: quien en Celaya voló el brazo derecho al general Álvaro Obregón. Chihuahua, México: Gobierno del estado de Chihuahua.

Hernández Orozco, G. (I998). Síntesis histórica de la Universidad Autónoma de Chihuahua. Chihuahua, México: UACH.

Hernández Orozco, G. (2004). Universidad Autónoma de Chihuahua. Chihuahua, México: UACH.

INEGI [Instituto Nacional de Estadística y Geografía]. (1950). Censo de población de los Estados Unidos Mexicanos de 1950. México: Instituto Nacional de Estadística y Geografía

UNAM. (1959). Anuario de Estadística 1959. Departamento de estadística. México: Universidad Nacional Autónoma de México.

Este artículo se publica bajo una licencia de Creative Commons Reconocimiento-NoComercial 4.0
Internacional, y puede ser usados gratuitamente para fines no comerciales, dando los créditos a los
autores y a la revista.

